\title{
Progestogens in menopausal hormone therapy
}

\author{
Małgorzata Bińkowska ${ }^{1}$, Jarosław Woroń2 \\ ${ }^{1} 1^{\text {st }}$ Department of Obstetrics and Gynaecology, Centre of Postgraduate Medical Education, Warsaw, Poland \\ 2Department of Clinical Pharmacology, Chair of Pharmacology, Faculty of Medicine, Jagiellonian University Medical College, Cracow, \\ Poland
}

\begin{abstract}
Progestogens share one common effect: the ability to convert proliferative endometrium to its secretory form. In contrast, their biological activity is varied, depending on the chemical structure, pharmacokinetics, receptor affinity and different potency of action. Progestogens are widely used in the treatment of menstrual cycle disturbances, various gynaecological conditions, contraception and menopausal hormone therapy. The administration of progestogen in menopausal hormone therapy is essential in women with an intact uterus to protect against endometrial hyperplasia and cancer. Progestogen selection should be based on the characteristics available for each progestogen type, relying on the assessment of relative potency of action in experimental models and animal models, and on the indirect knowledge brought by studies of the clinical use of different progestogen formulations. The choice of progestogen should involve the conscious use of knowledge of its benefits, with a focus on minimizing potential side effects. Unfortunately, there are no direct clinical studies comparing the metabolic effects of different progestogens.
\end{abstract}

Key words: progestogens, menopausal hormone therapy, progesterone, progestin.

\section{Introduction}

Progestogens are substances exhibiting a progestagenic effect, i.e. inducing secretory transformation in the endometrium which was previously affected by oestrogens, thus mimicking the activity of endogenous progesterone in the luteal phase of the menstrual cycle.

Although progestogens are currently widely used in gynaecology, and not uncommonly also in obstetrics, the body of knowledge about progestogens, as well as terminology, appears rather chaotic and insufficiently systematized. A variety of interchangeable names are used, including progestagen, progestogen, gestagen, gestogen and progestin. According to the recommendations issued by the North American Menopause Society, the present study uses the term "progestogen", by analogy to other classes of hormonal drugs, e.g. oestrogens or androgens, and the term "progestin" which refers to synthetic progestogens, i.e. excludes progesterone [1].

Progestogens produce their physiological and biological effects via the progesterone receptors $A$ (PR-A) and $B$ (PR-B). Both of them are nuclear receptors, the difference being that PR-B has an additional amino acid sequence at the terminal $\mathrm{NH}_{2}$ group. In experimental conditions progestins bind to both progesterone receptors with a high, though varied, affinity. The expression of genes by PR is regulated through different, also extragenomic, mechanisms. In addition, progestogens modulate gene transcription due to their affinity to other steroid receptors: androgen receptor (AR), glucocorticoid receptor (GR) and mineralocorticoid receptor (MR). Studies of affinity, as well as the type and relative potency of action of different progestogens, are based on experiments conducted on animal models and human cell lines and tissues. However, biological response may differ depending on the achieved concentration, duration of action and effects caused by adjacent structures and tissues.

The biological activity of progestogens is varied and influenced by their chemical structure, pharmacokinetics and potency of action. The metabolism of progestogens is still poorly understood, however it is definitely determined by the route of administration, among other factors. Following oral administration, progestogens are partially metabolized by enzymes produced by bacteria living in the stomach and small intestine, and during the process of mucosal absorption. After reaching the liver, the "first passage effect" is observed, i.e. an intensive metabolic process involving primarily cytochrome P450 enzymes. The liver is the main site for the metabolism of progestogens also when they are administered via other routes. Some progestins (e.g. desogestrel or norgestimate) are pro-drugs, i.e. become active only through the formation of metabolites. Finally, due to the activity of sulphatases, some conjugated 
metabolites may be subject to secondary absorption in the intestine and repeated activity.

Indications for progestogen treatment in the premenopausal period are menstrual disorders affecting both the frequency of the menstrual cycle (polymenorrhea, oligomenorrhea) and the severity of bleeding (hypomenorrhea, hypermenorrhea), and heavy prolonged bleeding without an underlying organic condition. Some progestins are used as contraceptives, either alone or in conjunction with oestrogen.

The only indication for progestogen use during menopausal hormone therapy (MHT) is to protect the uterus from endometrial hyperplasia and thus eliminate the risk of endometrial cancer which would be induced by the prolonged use of unbalanced oestrogens [2].

The selection of progestogens for therapy should be based on the available knowledge of their mechanisms of action and differences between them. The bioavailability of different progestogens depends on multiple factors including the route of administration, metabolism, protein-binding strength and additional binding to other steroid receptors (AR, GR, MR) and action triggered via this pathway. Progestogens vary in their activity (not only as a result of differences in chemical structure but also in the strength of binding to different receptors), intracellular activity and often also biological and clinical effects. What is more, they may exhibit differences in the safety profile and the risk of interactions with other drugs used concomitantly in combination therapy.

Another important factor affecting the pharmacokinetics of all drugs, progestogens included, is the aging process. Changes arise as a result of deteriorated liver function (impairment of the cytochrome P450 system, modified activity of P-glycoprotein) and kidney function, but also reduced cardiac output and decline in lung function. Another factor which merits consideration is the reduction of conjugation processes occurring in the liver, which is important for the metabolism and clearance of certain progestogens. It is usually accompanied by changes in body weight and composition, and aging-related decreased muscle mass. The factors should be taken into account in differentiating treatment and drug doses between perimenopausal and elderly women (over 65 years of age).

\section{Progesterone}

Progesterone $(P)$ triggers secretory transformation of the endometrium during the proliferative phase induced by oestradiol. It is critical for embryo implantation and maintenance of pregnancy. In addition to regulating the reproductive system, progesterone has a crucial effect on the mammary glands and the central nervous system.

It demonstrates a strong progestagenic and antioestrogenic activity in the endometrium and cervix, and exerts marked antimineralocorticoid and antiandrogenic effects as a result of competitive inhibition of $5 \alpha$-reductase activity in the skin.

After oral administration $\mathrm{P}$ is rapidly metabolized in the gastrointestinal tract and liver, producing up to 30 metabolites. Among them, $5 \alpha$ - and $5 \beta$-pregnanolones have a sedative effect through binding to the $G_{A B A}$ (gamma-aminobutyric acid type $A$ ) receptor. Other important metabolites include 20-dihydroprogesterone (25-50\% of progestogen potency), 11-deoxycorticosterone (strong mineralocorticosteroid), 17 $\alpha$-hydroxyprogesterone and the inactive end product - pregnanediol. The proportions of metabolites show a very high degree of individual variation. Low bioavailability after oral administration was improved by micronization and then encapsulation of micronized progesterone suspended in oil in gel capsules. Following oral administration of $200 \mathrm{mg}$ of progesterone in this form, the maximum P concentration determined by RIA (radioimmunoassay) occurs after 4 hours and amounts to $12 \mathrm{ng} / \mathrm{ml}$, the concentration of $5 \alpha$-pregnanolon is $30 \mathrm{ng} / \mathrm{ml}$ and $5 \beta$-pregnanolon $-60 \mathrm{ng} / \mathrm{ml}$. Approximately $17 \%$ of circulating $P$ is bound with a high affinity to CBG, and $80 \%$ is bound with low affinity to albumins.

Oral micronized progesterone (MP) preparations are used in MHT at daily doses of 200-300 mg, ensuring sufficient protection against endometrial hyperplasia with a standard oestrogen dose. In sequential therapy, combining MP at a dose of $200 \mathrm{mg}$ for 12-14 days with oral or transdermal oestrogen at a standard dose prevents endometrial hyperplasia, causing regular withdrawal bleeding in $60 \%$ of women and amenorrhoea in a part of the remaining group. MP at a lower dose (100 mg) administered for 21-25 days of the cycle in conjunction with $150 \mu \mathrm{g}$ of oestradiol via the transdermal route improved tolerance (reduced drowsiness, tiredness and dizziness), and increased the percentage of amenorrhoea.

The intramuscular form of MP in oil (50-100 mg daily), which is still available in some countries, is mainly used today in in vitro fertilization programmes. Clinical trials are currently under way investigating an aqueous solution for subcutaneous administration which, at a dose of $25 \mathrm{mg}$, would deliver a similar effect as intravaginal gel at a daily dose of $90 \mathrm{mg}$.

Intravaginal progesterone in the form of $8 \%$ gel (90 $\mathrm{mg})$ or tablets $(200 \mathrm{mg})$ is used after IVF up to 8-12 weeks, and is approved for the prevention of premature birth in patients with a history of premature termination of one previous pregnancy and shortening of the cervical length to $\leq 25 \mathrm{~mm}$. The intravaginal route of administration ensures higher and more stable $\mathrm{P}$ concentrations in the blood serum. After intravaginal application of $100 \mathrm{mg}$ of MP in a gel capsule, the maximum concentration of $P$ in the blood serum (around $5 \mathrm{ng} / \mathrm{ml}$ ) was noted after 6 hours, and was maintained 
for 24 hours. The concentration of $5 \alpha$-pregnanolone was $3.5 \mathrm{ng} / \mathrm{ml}$ after 2 hours, and the concentration of $5 \beta$-pregnanolone did not change [3].

A comparison of the effects of repeated administration of progesterone by the intramuscular $(2 \times 50 \mathrm{mg})$ and intravaginal $(4 \times 200 \mathrm{mg})$ routes reveals that the former route ensures a high constant concentration in the blood, whereas the latter causes a tenfold higher concentration in the uterus. The finding points to the so-called uterine first pass effect following intravaginal administration [4].

Transdermal progesterone preparations in the form of a cream or gel fail to ensure an adequate $P$ concentration in the blood despite achieving a variably high concentration in saliva and capillary blood tests. Consequently, in one third of cases the combination with 1 $\mathrm{mg}$ of oestradiol produces an inadequate endometrial effect: it remains in the proliferative phase or endometrial hyperplasia occurs [5]. It was assumed that the concentration of $P$ in the blood should exceed $5 \mathrm{ng} / \mathrm{ml}$ in order to inhibit mitotic divisions and induce the secretory transformation of the endometrium [6].

In some countries, $1 \%$ progesterone gel is approved for the treatment of premenstrual mastodynia. The topical administration of $P$ in the form of a cream or gel gives rise to concern because it leads to excessive tissue concentrations and concurrently low concentrations in the blood. Such supraphysiological saturation of tissues and progesterone receptors with progesterone may have an adverse effect on clinical response through the PR and a decrease in the ER, and cause an excessive formation of progesterone metabolites with agonist or antagonist activity [6]. New evaluated preparations of aqueous, rather than alcoholic, progesterone gels require in-depth clinical trials.

Attempts to use progesterone alone (without oestrogen) in the form of a cream at daily doses from 20 to $60 \mathrm{mg}$ to relieve vasomotor symptoms failed to improve the quality of life, and the achieved results did not differ from the placebo effect [7]. Progesterone has not been found to produce clinically significant interactions with other drugs. In vitro studies have shown that cytochrome P450 inhibitors, e.g. azole antifungal medications, can slow down the metabolism of progesterone. Progesterone may also increase the serum concentration of cyclosporine. High progesterone doses may transiently increase the rate of sodium and chloride excretion from the body.

\section{Review of progestins used in menopausal hormone therapy which are available in Poland}

\section{Dienogest}

Dienogest (DNG) is a derivative of 19-nortestosterone, but due to its structure it is the only pro- gestogen which also possesses the properties of $17 \alpha$-hydroxyprogesterone. This is because instead of having an ethyl group at position $\mathrm{C} 17$, dienogest has a cyanomethyl group and a double bond between C9 and $\mathrm{C} 10$. In addition to a very fast absorption rate, dienogest exhibits a very high bioavailability of around $90 \%$. It is bound to albumins in $90 \%$, and around $10 \%$ is in a free form. It binds neither to SHBG, nor CBG. Its metabolites are inactive and rapidly excreted. The half-life is 10 hours. Stable concentrations are achieved after two days of treatment. Dienogest does not accumulate in the body. It demonstrates a poor affinity to the PR, but has a very potent progestagenic effect in the endometrium, and causes endometrial atrophy after prolonged use. It shows antagonist activity by binding to the $A R$, and hence produces an antiandrogenic action equivalent to ca. $40 \%$ of the effect induced by cyproterone acetate. Dienogest does not interact with the GR, MR or ER.

In vivo, it has a powerful progestagenic and moderate antigonadotropic activity, without any androgenic, glucocorticoid or mineralocorticoid effects. A dose of $2 \mathrm{mg}$ inhibits the growth of ovarian follicles at $10 \mathrm{~mm}$ and maintains the concentration of progesterone at a low level, but has a weak inhibitory effect on FSH and $\mathrm{LH}$. Consequently, dienogest is believed to have a weak central antigonadotropic action, but a potent direct peripheral ovulation-inhibiting effect [8].

Dienogest in the form of $2 \mathrm{mg}$ tablets is used in the treatment of endometriosis. It has been available for many years in the same dose in a combined oral contraceptive pill (COCP) in conjunction with $30 \mu \mathrm{g}$ of ethinyloestradiol in cases where an additional indication is the treatment of moderate acne. Dienogest combined with oestradiol valerate in the first four-phasic COCP has an additional indication for the treatment of excessively heavy or prolonged menstrual bleeding.

In MHT, the same dose can be administered with $1 \mathrm{mg}$ or $2 \mathrm{mg}$ of oestradiol valerate.

Even high doses of the drug, more than ten times higher than the commonly used doses, do not exert an adverse effect on the lipid profile, coagulation system or carbohydrate metabolism. Dienogest is metabolized chiefly via the CYP3A4 isoenzyme. Drugs inhibiting the isoenzyme, including azole antifungals (ketoconazole, fluconazole, itraconazole), cimetidine, verapamil, macrolides (e.g. erythromycin, clarithromycin, roxythromycin), diltiazem, protease inhibitors (e.g. ritonavir, saquinavir, indinavir, nelfinavir), antidepressants (e.g. fluvoxamine, fluoxetin) and grapefruit juice, may decrease the clearance rate of dienogest and trigger adverse reactions. Drugs inducing the isoenzyme, such as phenytoin, barbiturates, primidone, carbamazepin, rifampicin, oxcarbazepine, topiramate, felbamate, griseofulvin, nevirapine and products containing St. John's wort (Hypericum perforatum), may increase the clear- 
ance rate of sex hormones and diminish the activity of dienogest, potentially causing changes in the uterine bleeding profile.

\section{Drospirenone}

Drospirenone (DRSP) is a derivative of $17 \alpha$-spironolactone. It is rapidly absorbed after oral administration, and reaches its maximum concentration in the blood within 1-2 hours. The bioavailability of DRSP is $76 \%$, and its half-life equals 25-33 hours. It is completely excreted within 10 days. Circulating DRSP is albumin-bound. It has no active metabolites. Its affinity to the mineralocorticoid receptor is estimated to be five times more potent than aldosterone. Consequently, it enhances the secretion of sodium and the retention of potassium with a dose-dependent intensity, approximately 8-10 times more potently than spironolactone. Drospirenone exhibits a more powerful antimineralcorticoid activity than progesterone. Through binding to the AR, it has an antiandrogenic effect whose relative potency corresponds to $30 \%$ of the standard (cyproterone acetate).

It is used in a combined oral contraceptive pill at a dose of $3 \mathrm{mg}$ in conjunction with 20 or $30 \mu \mathrm{g}$ of ethinyloestradiol.

Drospirenone has also found application in continuous low-dose MHT: $1 \mathrm{mg}$ of oestradiol is combined with $2 \mathrm{mg}$ of DRSP ensuring endometrial atrophy through the apoptotic activity on the glandular epithelium. The antimineralcorticosteroid action reduces salt retention and causes a statistically significant mild decline in systolic and diastolic blood pressure compared to placebo. DRSP $+E_{2}$ is also claimed to have a favourable effect on the sense of smell [9]. Drospirenone may affect the renin activity of plasma. It needs to be noted that patients taking drospirenone and angiotensinconverting-enzyme inhibitors, $\mathrm{AT}_{1}$ receptor antagonists and spironolactone may experience interactions manifested as an increase in blood serum concentration of potassium. Consequently, patients require potassium level monitoring during the initial period of treatment.

\section{Dydrogesterone}

Dydrogesterone is a retroprogesterone as a stereoisomer of progesterone with an additional double binding between C6 and C7. As a consequence, dydrogesterone differs from oral progesterone in its metabolism and action. Despite having a slightly lower affinity of binding to the PR, dydrogesterone exhibits better bioavailability, and doses necessary to achieve secretory transformation of the endometrium are 10-20 times lower. It has a highly selective action. It demonstrates no antigonadotropic activity and has no inhibitory effect on the ovulation. Also, dydrogesterone has no oes- trogenic, androgenic, antiandrogenic and glucocorticoid activity. It produces a weak antimineralcorticoid effect, but has no thermogenic properties. Dydrogesterone is highly active after oral administration. It is very rapidly absorbed and then metabolized, chiefly to $20 \alpha$-hydroxy derivatives including $20 \alpha$-dihydrodydrogesterone (DHD) which achieves the highest concentration after ca. 90 minutes and has a similar profile of action. The mean half-life of dydrogesterone is 5-7 hours, and DHD is 1417 hours. It is completely excreted within 72 hours. The equilibrium of concentrations is achieved after three days of treatment.

Dydrogesterone is used in all cases of deficiency of endogenous progesterone, but it does not have its thermogenic effect. At doses of 10-30 mg, dydrogesterone is used in the treatment of dysmenorrhoea, secondary amenorrhoea, irregular menstrual cycles, premenstrual syndrome, threatened or recurrent miscarriage, abnormal uterine bleeding and pain in endometriosis.

In sequential MHT, it has found application in two preparations in a $10 \mathrm{mg}$ course for 14 days in conjunction with oestradiol in $1 \mathrm{mg}$ or $2 \mathrm{mg}$ doses, and in continuous therapy in two consecutive regimens at $5 \mathrm{mg}$ or $2.5 \mathrm{mg}$ in conjunction with $1 \mathrm{mg}$ or $0.5 \mathrm{mg}$ of oestradiol, respectively. Such diversity of preparations makes it possible to adjust the type of MHT to the severity of menopausal symptoms and women's age. All of them ensure a statistically significant improvement in vasomotor symptoms and other observed menopausal symptoms in relation to the baseline - the onset of therapy - and comparable preparations [10]. There are no reports of clinically significant interactions of dydrogesterone with other concurrently taken drugs.

\section{Levonorgestrel and norgestrel}

Levonorgestrel (LNG) is a potent progestogen which does not have any glucocorticoid and antimineralcorticoid activity but displays a certain level of androgenic activity. DL-norgestrel is a mixture of equal parts of LNG and inactive D-norgestrel, hence the hormonal activity of $0.5 \mathrm{mg}$ of norgestrel is equivalent to $0.25 \mathrm{mg}$ of LNG.

The bioavailability of LNG after oral administration reaches $95 \%$. In the blood, it is bound in 50\% to albumins and in $48 \%$ to SHBG. The half-life is 24 hours and one hour, respectively. Due to its androgenic activity, it can lower the concentration of SHBG, unless (as in COCP) it is bound to a potent oestrogen - ethinyloestradiol (EE), cumulatively leading to the maintenance of the SHBG level. Levonorgestrel at a dose of $300 \mu \mathrm{g}$ used in conjunction with $2 \mathrm{mg}$ of oestradiol in postmenopausal women makes it possible to achieve the maximum concentration of $6.2 \mathrm{ng} / \mathrm{ml}$ after an hour, with a half-life of 32 hours.

The sequential transdermal release of $10 \mu \mathrm{g}$ of LNG in the second phase of the menstrual cycle and 
$50 \mu \mathrm{g}$ of oestradiol daily from a weekly patch ensures the mean blood concentration of LNG at a level of 120 $\mathrm{pg} / \mathrm{ml}$, and oestradiol at $30 \mathrm{pg} / \mathrm{ml}$. Other dose options (15 $\mu \mathrm{g}$ of LNG and $75 \mu \mathrm{g} \mathrm{E}_{2}$, and $20 \mu \mathrm{g}$ of LNG and $100 \mu \mathrm{g}$ of $E_{2}$ daily) also guarantee endometrial safety [11]. Continuous transdermal MHT, combining the release of $45 \mu \mathrm{g}$ of $E_{2}$ with various doses of LNG (15-30$45 \mu \mathrm{g})$ daily, has been shown to protect the endometrium from hyperplasia, but also cause irregular bleeding in a high percentage of cases (29-37\%).

The levonorgestrel intrauterine system LNG-IUS 20 is approved in some countries for endometrial protection during MHT. The vertical arm of the T-shaped system is provided with a reservoir containing $52 \mathrm{mg}$ of LNG. The initial release is $20 \mu \mathrm{g}$, and the mean release is $12 \mu \mathrm{g}$ of LNG daily for a period of 5 years. The LNG concentration in the blood is $0.5 \mathrm{ng} / \mathrm{ml}$ during the first year of use. After an initial couple of months of irregular bleeding, continuous LNG release leads to endometrial atrophy and stoppage of bleeding. In conjunction with $E_{2}$ administered orally at a daily dose of $2 \mathrm{mg}$ or $E_{2}$ released transdermally at a dose of $50 \mu \mathrm{g}$, it offers effective endometrial protection and halts bleeding in two thirds of postmenopausal women. A new smaller version of the system containing $13.5 \mathrm{mg}$ of LNG and releasing initially $14 \mu \mathrm{g}$ of LNG daily and $6 \mu \mathrm{g}$ on average is used as a contraceptive, however it has not as yet been investigated to determine its potential suitability for MHT. Barbiturates, phenytoin, products containing St. John's wort, ritonavir, griseofulvin, rifampicin, ampicillin and other antibiotics weaken the activity of levonorgestrel. Levonorgestrel-containing preparations may increase the risk of toxic activity of cyclosporine.

\section{Norethisterone and norethisterone acefote}

Norethisterone (NET) and norethisterone acetate (NETA) are characterized by very similar pharmacokinetics and pharmacodynamics. Following oral administration NETA is rapidly hydrolyzed to NET. Their bioavailability is $40-80 \%$ and depends on molecule size and time of taking the drug (it is higher for smaller molecules taken in the fasted state) [12]. In the blood, NET is bound in $60 \%$ to albumins and in $36 \%$ to SHBG. The half-life is 9.5 hours and 1.5 hours, respectively. Around $90 \%$ of metabolites retain the ethyl group. One of them, $5 \alpha$-dihydro-NET, has a high relative affinity to the $A R$ and is responsible for its weak androgenic activity. A trace amount of NET $(<0.5 \%)$ is aromatized in the liver to ethinyloestradiol, which has no clinical relevance at a dose of $1 \mathrm{mg}$, however $5 \mathrm{mg}$ of NET is equivalent to the ingestion of $30 \mu \mathrm{g}$ of EE [13]. Norethisterone demonstrates no glucocorticoid or antimineralcorticoid activity. After taking a single $0.5 \mathrm{mg}$ dose of NETA the maximum concentration of NET in the blood $(5 \mathrm{ng} / \mathrm{ml})$ is detected after one hour. For the $1 \mathrm{mg}$ dose, it equals
5-10 ng/ml, and for $2 \mathrm{mg}-12 \mathrm{ng} / \mathrm{ml}$. A combination with oestradiol does not alter the pharmacodynamics of NET. Multiple repeated doses ensure a stable concentration of ca. $7.4 \mathrm{ng} / \mathrm{ml}$.

The transdermal release of $250 \mu \mathrm{g}$ of NETA from a patch produces the concentration of $0.5-1.0 \mathrm{ng} / \mathrm{ml}$ on the second day of application, after which it falls to $0.25-0.5 \mathrm{ng} / \mathrm{ml}$. The continuous transdermal application of 140 or $250 \mu \mathrm{g}$ of NETA combined with $50 \mu \mathrm{g}$ of oestradiol provides effective endometrial protection from hyperplasia. The frequency of bleeding is lower at smaller NETA doses. Similarly, sequential therapy combining the release of $50 \mu \mathrm{g}$ of oestradiol with a 170 or $250 \mu \mathrm{g}$ addition of NETA between the $15^{\text {th }}$ and $28^{\text {th }}$ day of the cycle protects the endometrium and causes regular withdrawal bleeding in $80 \%$ of women, irregular bleeding in $11 \%$ and no bleeding in 9\% [14]. Rifampicin, some antiepileptic medicines (phenytoin), barbiturates and phenylbutazone accelerate the metabolism of norethisterone, thus weakening its progestogenic action.

\section{Cyproterone acetate}

Cyproterone acetate (CPA) is bioavailable in nearly $100 \%$ after oral administration. The majority of the drug (ca. 93\%) remains bound to albumins, without binding either to SHBG or CBG. It demonstrates the highest antiandrogenic activity of all progestins, chiefly as a result of competitive inhibition of androgen binding to the AR with a dose-dependent potency, and only to a minor extent as a consequence of activity exerted by the important metabolite $5 \beta$-hydroxyCPA. After a single $2 \mathrm{mg}$ dose CPA achieves the blood serum concentration of $11 \mathrm{ng} / \mathrm{ml}$. Cyproterone acetate accumulates in fatty tissues, which may lead to secondary disorders of the bleeding pattern during prolonged treatment and after discontinuation.

It is used in the therapy of hirsutism, androgenic alopecia, and severe and moderate androgen-induced acne which is not successfully treated with other antiandrogenic drugs. Cyproterone is metabolized by isoenzyme CYP3A4 of cytochrome P450, so its metabolism can be inhibited by ketoconazole, itraconazole, clotrimazole and ritonavir. On the other hand, CYP 3A4 inducers (e.g. rifampicin, phenytoin, products containing St. John's wort) can increase the metabolism of cyproterone, reducing its blood concentration. Cyproterone at high doses $(300 \mathrm{mg} / \mathrm{d})$ can inhibit isoenzymes CYP 2C9, 2C19, 3A4 and 2D6 of cytochrome P450, inducing the risk of adverse pharmacokinetic interactions with diverse clinical features. The concurrent use of statins (especially lovastatin, simvastatin and atorvastatin) increases the risk of myopathy or rhabdomyolysis. Consequently, muscle pain experienced by patients taking cyproterone acetate and statins should not be ignored. 


\section{Medroxyprogesterone acetate}

Medroxyprogesterone acetate (MPA) has a higher progestagenic activity and better bioavailability than oral progesterone thanks to a slightly different chemical structure (methyl group at C6 and acetate group at C17). Medroxyprogesterone acetate exhibits a relatively high affinity to the PR. It also binds to the androgen and glucocorticoid receptors, however without binding to the oestrogen and mineralcorticoid receptors.

Following oral administration, it binds weakly to albumins, but without binding to SHBG. In fact, it seems to decrease the concentration of $\mathrm{SHBH}$ by around a dozen percent owing to weak androgenic properties. After a single oral dose of $10 \mathrm{mg}$, MPA achieves the maximum concentration in the blood, in the range of 3.4-4.4 ng/ml, after 1-4 hours. The concentration falls rapidly after 6-12 hours, and equals $0.3-0.6 \mathrm{ng} / \mathrm{ml}$ after 24 hours [15].

The sequential administration of MPA in conjunction with $E_{2} V$ has been shown to cause a 1.6-1.8 increase in the AUC (area under the curve) in women over 65 years of age compared to younger postmenopausal women.

Medroxyprogesterone acetate is used in contraception (subcutaneous or intramuscular injections of longacting forms), in the treatment of excessively heavy or prolonged bleeding, for the relief of endometriosis pain and in MHT. Menopausal hormone therapy preparations which are available in Poland contain oestradiol valerate. However, there are no classic combinations with conjugated oestrogens which were investigated in the WHI (Women's Health Initiative) study, and previously in HERS I and II (Heart and Estrogen/Progestin Replacement Study). Some years ago, high-dose MPA therapy was administered in endometrial hyperplasia and inoperable endometrial cancer. The concurrent use of aminoglutethimide can accelerate the biological transformation of medroxyprogesterone and diminish
Tab. I. Classification of progestogens

\begin{tabular}{|c|}
\hline 1. Progesterone \\
\hline 2. Retroprogesterone (dydrogesterone) \\
\hline 3. Progesterone derivatives \\
\hline A. $17 \alpha$-hydroxyprogesterone derivatives (pregnanes) \\
\hline $17 \alpha$-hydroxyprogesterone caproate \\
\hline $17 \alpha$-hydroxyprogesterone heptanoate \\
\hline Chlormadinone acetate \\
\hline Cyproterone acetate \\
\hline Medroxyprogesterone acetate \\
\hline B. 19-norprogesterone derivatives (norpregnanes) \\
\hline Nomegestrol acetate \\
\hline Demegestone \\
\hline Promegestone \\
\hline Trimegestone \\
\hline Nesterone \\
\hline 4. Testosterone derivatives \\
\hline A. 19-nortestosterone derivatives (estranes) \\
\hline Lynestrenole \\
\hline Norethynodrel \\
\hline Norethisterone/norethisterone acetate \\
\hline Ethynodiol diacetate \\
\hline Norgestrinone \\
\hline Dienogest \\
\hline B. 19-nortestosterone derivatives (13-ethylgonanes) \\
\hline Levonorgestrel / norgestrel \\
\hline Desogestrel / etonogestrel (active metabolite) \\
\hline Gestodene \\
\hline Norgestimate/norelgestromin (active metabolite) \\
\hline 5. Spironolactone derivatives \\
\hline Drospirenone \\
\hline
\end{tabular}

Tab. II. Biological activity of progestogens

\begin{tabular}{|c|c|c|c|c|c|c|c|c|}
\hline Progestogen type & $\begin{array}{l}\text { Progesto- } \\
\text { genic }\end{array}$ & $\begin{array}{l}\text { Antigon- } \\
\text { adotropic }\end{array}$ & $\begin{array}{l}\text { Antioes- } \\
\text { trogenic }\end{array}$ & $\begin{array}{l}\text { Oestro- } \\
\text { genic }\end{array}$ & $\begin{array}{l}\text { Andro- } \\
\text { genic }\end{array}$ & $\begin{array}{l}\text { Antiandro- } \\
\text { genic }\end{array}$ & $\begin{array}{l}\text { Glucocor- } \\
\text { ticoid }\end{array}$ & $\begin{array}{l}\text { Antimin- } \\
\text { eralocorti- } \\
\text { coid }\end{array}$ \\
\hline Progesterone & + & + & + & - & - & $+/-$ & + & + \\
\hline Dienogest & + & + & $+/-$ & $+/-$ & - & + & - & - \\
\hline Drospirenone & + & + & + & - & - & + & - & + \\
\hline Dydrogesterone & + & - & + & - & - & $+/-$ & - & $+/-$ \\
\hline Levonorgestrel & + & + & + & - & + & - & - & - \\
\hline Norethisterone & + & + & + & + & + & - & - & - \\
\hline Cyproterone acetate & + & + & + & - & - & ++ & + & - \\
\hline $\begin{array}{l}\text { Medroxyprogesterone } \\
\text { acetate }\end{array}$ & + & + & + & - & $+/-$ & - & + & - \\
\hline Nomegestrol acetate & + & + & + & - & - & $+/-$ & - & - \\
\hline
\end{tabular}


its efficacy. The drug can also interfere with metyrapone test results.

\section{Nomegestrol acetate}

Nomegestrol acetate (NOMAC) is a derivative of 19-norprogesterone. It achieves its maximum blood concentration within 2-3 hours after oral administration irrespective of the dose, and has a half-life of 50 hours. The bioavailability of the drug is $65 \%$. NOMAC is metabolized in the liver, mainly in the CYP3A4 and CYP3A5 systems, which is why it can enter into clinically significant interactions under the influence of ketoconazole, rifampicin and certain anticonvulsant drugs. Anticonvulsants (carbamazepine, phenobarbital, phenytoin, primidone), barbiturates, griseofulvin, rifabutin and rifampicin can decrease the efficacy of nomegestrol [16, 17]. Stable concentrations are achieved after five days of treatment. Impaired liver function can result in the accumulation of the drug. The majority of the drug binds to albumins, without binding to SHBG and CBG [18]. It is a very potent PR agonist. It demonstrates a weak antioestrogenic action, and does not bind to the AR, GR or MR.

Nomegestrol acetate is used at daily doses ranging from 2.5 to $10 \mathrm{mg}$ in the management of menstrual disorders.

Due to its potent antigonadotropic action, nomegestrol acetate is used in COCP at a dose of $2.5 \mathrm{mg}$ in conjunction with oestradiol $(1.5 \mathrm{mg})$ in the regimen of 24 active tablets plus 4 placebo tablets.

In MHT applications, studies were conducted on regimens combining NOMAC at doses of $1.5-5 \mathrm{mg}$ with oestradiol at doses of 1.0-2.5 mg. The studies demonstrated the elimination of menopausal symptoms

Tab. III. Daily oral progestogen dose ensuring endometrial protection in MHT [19]

\begin{tabular}{lcc}
\hline Progestogen & \multicolumn{2}{c}{ Daily dose (mg) } \\
\cline { 2 - 3 } & $\begin{array}{c}\text { Therapy } \\
\text { Sequential }\end{array}$ & Continuous \\
\hline Progesterone & $200-300$ & 100 \\
\hline Dienogest & $3-4$ & - \\
\hline Drospirenone & - & 2 \\
\hline Dydrogesterone & $10-20$ & $2.5-10$ \\
\hline Levonorgestrel & $0.075-0.15$ & - \\
\hline Cyproterone acetate & 1 & - \\
\hline Medroxyprogesterone acetate & $5-10$ & 2.5 \\
\hline Nomegestrol acetate & $5-10$ & 2.5 \\
\hline Norethisterone acetate & $1-2$ & $0.5-1.0$ \\
\hline
\end{tabular}

$(-)$ symbol - not determined. accompanied by a concurrent favourable effect on blood lipids, but an increase in the concentration of antithrombin III.

Nomegestrol acetate is used in sequential therapy at a dose of $5 \mathrm{mg}$ for 12-14 days of the cycle, and in continuous therapy at 2.5-3.75-5 mg daily.

\section{Clinical activities of progestogens in menopausal hormone therapy}

\section{Effect on the endometrium}

The main and essential activity exerted by progestogen is the transformation of the endometrium from its proliferative into secretory form, and hence endometrial protection from hyperplasia and cancer. The antiproliferative action stems from the inhibition of oestrogeninduced mitotic activity, ER reduction, increased activity of enzymes (17 $\beta$-hydroxysteroid dehydrogenase type 2 converting oestradiol into oestrone, and sulphotransferase leading to the formation of oestrone sulphate), which results in oestrogen elimination from the body and IGFBP-1 stimulation inhibiting the mitogenic effect of the IGF on the endometrium.

The optimum daily progestogen dose should inhibit mitotic activity within a short period ( $\leq 2$ mitoses $/ 1,000$ glandular cells at the end of a single course of treatment), and should not induce endometrial hyperplasia over a long period ( $\leq 2$ cases of hyperplasia/100 women/12 months). In addition to the dose, a significant factor is also the duration of use (at least 10 days in cyclic treatment and 12 days in sequential therapy).

The best endometrial protection is achieved with the continuous therapeutic regimen. Table III lists oral progestogen doses used in MHT.

\section{Effect on the mammary glands}

A recent publication presenting results obtained in the 13-year follow-up of women who took part in the Women's Health Initiative (WHI) study demonstrated that the total relative risk (RR) of breast cancer was 1.28 (1.11-1.48) in oestrogen/progestogen therapy, and 0.79 (0.65-0.97) in oestrogen monotherapy. The values were statistically significant in both cases, suggesting that the increase or decrease in the risk of breast cancer was crucially dependent on the presence of progestogen (in this case medroxyprogesterone acetate) [20]. Nevertheless, interpretations of the results are very cautious both in terms of risk $(<1$ breast cancer case per 1,000 women more in the group receiving oestrogen/progestogen therapy) and protection ( $<1$ breast cancer case per 1,000 women less in the oestrogen therapy group). Commentators draw attention to the possibility of systematic errors, including the bias of detection or monitoring, which may eliminate any un- 
favourable or favourable effect of therapy [21]. However, results of other epidemiological studies published after 2000 indicate consistently that adding progestin to oestrogen contributes to an increase in breast cancer risk compared to oestrogen monotherapy. For example, a study of a population of 33,000 women aged 50 to 64 years showed that the risk of breast cancer in users of oestrogens alone was OR $=0.96(0.88-1.06)$, but in users of conjugated $\mathrm{E}+\mathrm{P}$ it rose to $\mathrm{OR}=1.44(1.31-1.58)$ [22]. Similar outcomes were recorded in a cohort study of 67,754 women. In the group using E + P therapy, there was an increased risk of ductal cancer $-R R=1.75$ (1.59-2.01) and lobular breast cancer $-\mathrm{RR}=2.12$ (1.62$2.77)$, whereas in the group taking oestrogens only (CEE) the values were: $R R=0.99$ (0.84-1.17) for ductal cancer and $R R=1.13$ (0.94-1.78) for lobular breast cancer [23]. Important findings were also reported in the French E3 Cohort Study, indicating that not all progestogens act in the same way, and that the outcomes of the WHI study should not be extrapolated to other MHT types and regimens [24]. In a group of 80,377 postmenopausal women, of which $70 \%$ used MHT for an average of 7 years and were followed up for 8 years, there were 2,354 cases of breast cancer. No statistically significant increase in the risk of breast cancer was found among women using transdermal oestrogens alone and oestrogens in conjunction with progesterone or dydrogesterone during up to 5 years of therapy. In contrast, a statistically significant increase in breast cancer risk was determined for other $\mathrm{E}+\mathrm{P}$ therapy regimens containing seven other progestogens: chlormadinone acetate, cyproterone acetate, medroxyprogesterone acetate, nomegestrol acetate, norethisterone acetate, promegestone or medrogestone. The route of oestrogen administration was also shown to be a potentially important factor.

The highest proliferative activity in normal breast tissue in the physiological cycle is observed in the luteal phase. Experimental in vitro studies aim to evaluate the effect of different progestogens on apoptosis/ proliferation indices in healthy tissue cell lines and in breast cancer. Unfortunately, different progestogens are rarely investigated using the same model of experimental conditions and breast cell lines.

\section{Effect on the cardiovascular system}

Evaluation of the impact of MHT on the risk of cardiovascular diseases takes into account effects on the composition of blood lipids, arterial function, vascular inflammatory markers, changes in the blood coagulation system and carbohydrate metabolism.

The WHI study concerning both the entire investigated group of women and detailed analyses of therapy in consecutive decades of life point to major differences between the oestrogen arm (CEE) and the oestrogen/ progestogen arm (CEE + MPA) in favour of the former. An example is the frequency of cardiac episodes reported among women in the age group of 50-59 years. In the group receiving oestrogen therapy the risk was lower $(H R=0.63(0.36-1.09))$ than in the group of $E+P$ therapy $(H R=1.29(0.79-2.12))$ [25]. Similar differences were noted for the risk of venous thromboembolism. Combined $E+P$ therapy caused a significant increase in the risk of thromboembolic complications: $\mathrm{HR}=2.06$ (1.57-2.70), whereas in the oestrogen arm only a growing trend was noted: $\mathrm{HR}=1.33$ (0.86-2.08) [26]. A question arises as to the degree to which the findings are attributable to medroxyprogesterone acetate and the role of other factors, e.g. different characteristics of the compared women's cohorts.

An adverse effect of MPA was already noted in the first randomized clinical trial of MHT: Postmenopausal Estrogen/Progestin Intervention (PEPI). A significantly higher rise in HDL cholesterol concentration was only noted in the group which received P. In macaque studies, CEE + MPA increased fasting glucose and insulin levels, and led to worse glucose challenge test results than in the group which only received CEE.

A positive effect on the blood lipids is noted for progesterone and progestins - 19-norprogesterone derivatives, as opposed to a negative effect induced by 19-nortestosterone and MPA.

A metaanalysis of 248 studies published in 19742000, encompassing 42 different MHT regimens, enabled an assessment of progestogens focusing on their impact on the lipid profile [27]. In general terms, progesterones modified the oestrogen-induced fall in total and LDL cholesterol levels only to a minor degree, and as a rule had no impact on the adverse rise in triglycerides triggered by oral oestrogen intake. Based on the elimination of the beneficial rise in HDL cholesterol concentration, they can be ranked as follows: dydrogesterone and medrogestone - the lowest effect, followed by progesterone, CPA, MPA, transdermal NETA, LNG and oral NETA.

C-reactive protein (CRP) is recognized as a marker of chronic arteritis which is linked to the risk of coronary heart disease, ischaemic stroke and vascular mortality. The importance of different oestrogen effect on the CRP value depending on the route of administration and dose is debatable. Progestins which demonstrate a certain level of androgenic activity are able to influence the concentration of CRP, lipid profile and glucose tolerance. An addition of progesterone or dydrogesterone to oestrogen does not negatively affect the CRP concentration [28].

In the group of progestogens, drospirenone is distinct for its statistically significant effect on the decrease in morning systolic blood pressure caused by its antimineralcorticoid properties [29]. A beneficial effect on 24-h ambulatory arterial blood pressure measure- 
ments in healthy women is achieved through the combination of a low dose $(1 \mathrm{mg})$ of oestradiol with dydrogesterone [30].

Progesterone and some pregnanes, devoid of androgenic activity, seem to have a beneficial effect on the parameters of the coagulation system. The observation was corroborated in the prospective cohort study E3N (Etude Epidémiologique de l'Education Nationale) which analyzed a total of 549 cases of first venous thromboembolism in a group of 80,308 postmenopausal women [31]. Neither progesterone nor pregnanes such as dydrogesterone and chlormadinone acetate were found to affect the risk of venous thromboembolism. Norpregnanes (nomegestrol acetate and promegestone) led to a significant increase in the risk of venous complications: $\mathrm{OR}=1.8$ (1.2-2.7).

A metaanalysis of studies addressing the effects of MHT on the metabolic syndrome showed MHT to reduce insulin resistance in healthy and diabetic women [32]. Dydrogesterone does not cancel out the favourable impact of oestradiol on fasting glucose and insulin concentrations, and even halts the menopause-related trend for disorders of insulin secretion [33].

\section{Effects on bones}

The majority of progestogens do not interfere with the favourable activity of oestrogens in the prevention and treatment of osteoporosis.

Only norethisterone acetate in high doses, which are unacceptable nowadays due to their adverse vascular effects, would be capable of preventing postmenopausal bone resorption alone [34].

\section{Effects on the brain}

Progesterone is an important neurosteroid which is synthesized de novo in the brain, spinal cord and peripheral nerves. In clinical trials it exhibits neuroprotective properties after brain injury [35].

Widely known experimental studies conducted by Catherine Woolley and Elizabeth Gould showed progesterone to inhibit, within a period of 12 hours, the process of oestradiol-induced synapse formation in the rat hippocampus. The effect occurs even though the presence of the classic PR was not identified, however its expression was detected in extranuclear neuronal sites [36].

In turn, cultures of rat hippocampus neurons, in which progesterone and 19-norprogesterone had a protective activity, showed that MPA exerted an opposite effect, eliminating the positive effects produced by oestradiol. Medroxyprogesterone acetate also antagonizes the restoration of mitochondrial function provoked by oestradiol and progesterone in ovariecto- mized rat females [37]. There is, as yet, no basis for generalizing conclusions arising from scarce experimental studies.

\section{Conclusions}

For many decades, the role of progesterone in MHT was limited to endometrial protection. There is an increasing body of data coming both from experimental studies and clinical trials, suggesting not only that there are significant dissimilarities between different progestogens, but also that they can variously modify the actions induced by oestrogens. Gaining greater insights into their role should make it possible to consciously adjust progestogen use during MHT.

\section{References}

1. North American Menopause Society. Role of progestogen in hormone therapy for postmenopausal women: Position Statement of the North American Menopause Society. Menopause 2004; 10: 113-132.

2. Bińkowska M, Dębski R, Paszkowski T, et al. Rekomendacje Polskiego Towarzystwa Menopauzy i Andropauzy na temat hormonalnej terapii menopauzy - stan wiedzy na grudzień 2013 roku. Prz Menopauzalny 2014; 13: 1-8.

3. Nahoul K, Dehennin L, Jondet M, et al. Profiles of plasma estrogens, progesterone and their metabolites after oral and vaginal administration of estradiol or progesterone. Maturitas 1993; 16: 185-202.

4. Ruan X, Mueck AO. Systemic progesterone therapy - oral, vaginal, injection and even transdermal? Maturitas 2014; 79: 248-255.

5. Wren BG. Progesterone creams: do they work? Climacteric 2003; 6: 184-187.

6. Stanczyk FZ. Treatment of postmenopausal women with topical progesterone creams and gels: are they effective? Climacteric 2014; 17 (Suppl 2): 8-11.

7. Whelan AM, Jurgens JM, Trinacty M. Bioidentical progesterone cream for menopause-related vasomotor symptoms. Ann Pharmacother 2013; 47: 112-116.

8. Ruan X, Seeger H, Mueck AO. The pharmacology of dienogest. Maturitas 2012; 71: 337-344.

9. Caruso S, Serra C, Grillo C, et al. Prospective study evaluating olfactometric and rhino manometric outcomes in postmenopausal women in $1 \mathrm{mg} 17 \beta$-estradiol and $2 \mathrm{mg}$ drosperinone HT. Menopause 2008; 15 : 967-972.

10. Stevenson JC, Durand G, Kahler E, et al. Oral ultra-low dose continuous combined hormone replacement therapy with $0.5 \mathrm{mg} 17 \beta$-oestradiol and $2.5 \mathrm{mg}$ dydrogesterone for the treatment of vasomotor symptoms: results from a double-blind, controlled study. Maturitas 2010; 67: 227232.

11. Sturdee DW, van de Weijer P, van Holst T, et al. Endometrial safety of a transdermal sequential estradiol-lewonorgestrel combination. Climacteric 2002; 5: 170-177.

12. Boyd RA, Zegarac EA, Eldon MA. The effect of food on the bioavalilability of norethindrone and ethynyl estradiol from norethindrone acetale/ ethynyl estradiol tablets intended for continuous hormone replacement therapy. Clin Pharmacol 2003; 43: 52-58.

13. Kuhnz W, Heuner A, Hümpel M, et al. In vivo transformation of norethisterone and norethisterone acetate to ethinyl estradiol in postmenopausal women. Contraception 1997; 56: 379-385.

14. Lindgren R, Risberg B, Hammar M, et al. Endometrial effects of transdermal estradiol/norethisterone acetate. Maturitas 1992; 15: 71-78.

15. Hiroi M, Stanczyk FZ, Goebelsmann PF, et al. Radioimmunoassay of serum medroxyprogesterone acetate (Provera) in women following oral and intravaginal administration. Contraception 1975; 26: 373-378.

16. Kostka-Trąbka E, Woroń J. Interakcje leków w praktyce klinicznej. Wydawnictwo Lekarskie PZWL, Warszawa 2011. 
17. Hanslen PD, Horn JR. Top 100 Drug Interactions 2015. H\&H Publications, Freeland 2015.

18. Ruan X, Seeger H, Mueck AO. The pharmacology of nomegestrol acetale. Maturitas 2012; 71: 345-353.

19. Schindler AE, Campagnoli C, Druckmann R, et al. Classification and pharmacology of progestins. Maturitas 2003; 46 Suppl 1: S7-S16.

20. Manson JE, Chlebowski RT, Stefanick ML, et al. Menopausal therapy and health outcomes during the intervention and postintervention and extended poststopping phases of the Women's Health Initiative randomized controlled trials. JAMA 2013; 310: 1353-1368.

21. Shapiro S. Risk of breast cancer among users of estrogen plus progestagen and unopposed estrogen: is the likelihood of bias symmetrical? Climacteric 2015; 18: 1-3.

22. Jick SS, Hagberg KW, Kaye JA, Jick H. Postmenopausal estrogen-containing hormone therapy and the risk of breast cancer. Obstet Gynecol 2009; 113: 74-80.

23. Calle EE, Feilgelson HS, Hildebrand JS, et al. Postmenopausal hormone use and breast cancer associations differ by hormone regimen and histologic type. Cancer 2009; 115: 936-455.

24. Fournier A, Berrino F, Riboli E, et al. Breast cancer risk in relation to different types of hormone replacement therapy in the E3N-EPIC kohort. Int J Cancer 2005; 114: 448-454.

25. Rossouw JE, Prentice RL, Manson JE, et al. Postmenopausal hormone therapy and risk of cardiovascular disease by age and years since menopause. JAMA 2007; 297: 1465-1477.

26. Anderson GI, Limacher M, Assaf AR, et al. Effects of conjugated equine estrogen in postmenopausal women wuthhysterectomy: the Women's Health Initiative randomized controlled trial. JAMA 2004; 291: 1701 1712.

27. Godsland IF. Effects of postmenopausal hormone replacement therapy on lipid, lipoprotein, and apolipoprotein (a) concentrations: analysis of studies published from 1974-2000. Fertil Steril 2001; 75: 898-915.
28. Bray PF, Larson JC, Lacroix AZ, et al. Usefulness of baseline lipids and C-reactive protein in women receiving menopausal hormone therapy as predictors of treatment-related coronary events. Am J Cardiol 2008; 101: 1599-1605.

29. White WB, Hanes V, Mallareddy M, Chauhan V. Effects of hormone therapy, drospirenone and $17 \beta$-estradiol, on early morning blood pressure in postmenopausal women with hypertension. J Am Soc Hypertens 2008; 2: 20-27.

30. Kaya C, Cengiz SD, Cengiz B, et al. Long-term effects of low-dose $17 \beta$-estradiol plus dydrogesterone on 24-h ambulatory blood pressure in healthy postmenopausal women: a 1-year, randomized, prospective study. Gynecol Endocrinol 2007; 23 (Suppl 1): 62-67.

31. Canonico M, Fournier A, Carcaillon L, et al. Postmenopausal hormone therapy and risk of idiopathic venous thromboembolism. Results from the E3N cohort study. Arterioscler Thromb Vasc Biol 2010; 30: 340-345.

32. Salpeter SR, Walsh JM, Ormiston TM, et al. Meta-analysis: effect of hormone replacement therapy on components of the metabolic syndrome in postmenopausal women. Diabetes Obes Metab 2006; 8: 538-554.

33. Stevenson JC, Panay N, Pexman-Fieth C. Oral estradiol and dydrogesterone combination therapy in postmenopausal women: review of efficacy and safety. Maturitas 2013; 76: 10-21.

34. Abdalla HI, Hart DM, Lindsay R, et al. Prevention of bone mineral loss in postmenopausal women by norethisterone. Obstet Gynecol 1985; 66: 789-792.

35. Stein DG, Wright DW. Progesterone in the clinical treatment of acute traumatic brain injury. Expert Opin Investig Drugs 2010; 19: 847-857.

36. McEwen BS. Sex, stress and the brain: interactive actions of hormones on the developing and adult brain. Climacteric 2014; 17 (Suppl 2): 18-25.

37. Irvin RW, Yao J, Ahmed SS, et al. Medroxyprogesterone acetate antagonizes estrogen up-regulation of brain mitochondrial function. Endocrinology 2011; 152: 556-567. 